\title{
Relationship between severity of obsessive- compulsive symptoms and schizotypy in obsessive-compulsive disorder
}

This article was published in the following Dove Press journal:

Neuropsychiatric Disease and Treatment

5 December 2012

Number of times this article has been viewed

\section{Haruka Yamamoto' \\ Hideto Tsuchida' \\ Takashi Nakamae' \\ Seiji Nishida' \\ Yuki Sakai' \\ Akihito Fujimori' \\ Jin Narumoto' \\ Yoshihisa Wada' \\ Takafumi Yoshida ${ }^{2}$ \\ Chiaki Taga ${ }^{3}$ \\ Kenji Fukui'}

'Department of Psychiatry, Graduate School of Medical Science, Kyoto Prefectural University of Medicine, Kyoto, Japan; ${ }^{2}$ Kyoto Cognitive Behavior Therapy Counseling Room, Kyoto, Japan; ${ }^{3}$ Department of Psychiatry, Kyoto Second Red Cross Hospital, Kyoto, Japan
Correspondence: Haruka Yamamoto Department of Psychiatry, Graduate School of Medical Science, Kyoto Prefectural University of Medicine, 465 Kajii-cho, Kawaramachi-Hirokoji, Kamigyo-ku, Kyoto 602-8566, Japan

$\mathrm{Tel}+8 \mathrm{I} 7525 \mathrm{I} 56 \mathrm{I} 2$

Fax +8I 75 25। 5839

Email y-haruka@koto.kpu-m.ac.jp
Purpose: Obsessive-compulsive disorder (OCD) patients exhibit a noninhibition response pattern very similar to that observed in schizotypy patients in cognitive tasks. It has been suggested that the reduced cognitive inhibition observed in both schizotypy and OCD may result in the frequent entry into awareness of unacceptable urges and intrusive thoughts. The aim of this study was to investigate the relationship between the severity of obsession or compulsion and schizotypy in OCD.

Patients and methods: Sixty subjects ( 25 males and 35 females) who were OCD outpatients in the University Hospital at the Kyoto Prefectural University of Medicine during the period 2008-2010 were enrolled in the study. Assessments of these patients were made using the Yale-Brown Obsessive Compulsive Scale (Y-BOCS), the Schizotypal Personality Questionnaire (SPQ), the Hamilton Depression Rating Scale (HAM-D), and the Hamilton Anxiety Rating Scale (HAM-A). The Pearson correlation coefficients between Y-BOCS and SPQ scores were calculated. Furthermore, hierarchical multiple linear regression analyses were conducted to assess whether schizotypy predicted the severity of obsession and compulsion.

Results: By calculating the Pearson correlation coefficient, it was found that the Y-BOCS obsession score, not the Y-BOCS compulsion score, was correlated with the SPQ total score. Results of the hierarchical multiple linear regression analysis showed that SPQ total score was a significant predictor of the Y-BOCS obsession score, after accounting for control variables (ie, HAM-D and HAM-A).

Conclusion: Results of this study showed that the Y-BOCS obsession score, not the Y-BOCS compulsion score, was correlated with the SPQ total score. This finding suggests that OCD patients with an elevated SPQ total score experience a reduction of cognitive inhibition, resulting in the frequent entry into obsession. Future longitudinal studies are recommended to clarify the effect of schizotypy on the clinical course of OCD.

Keywords: Y-BOCS, obsession, compulsion, severity

\section{Introduction}

Obsessive-compulsive disorder (OCD) has a lifelong prevalence rate of approximately $2.5 \%$ in the general population. ${ }^{1}$ Sobin et $\mathrm{al}^{2}$ have suggested that about half of OCD patients exhibit moderate to severe levels of schizotypy, and the authors have also identified a subtype of OCD with specifically high schizotypy. The term "schizotypy" derives from the abbreviated form of "schizophrenic phenotype" (ie, "schizotype"). ${ }^{3}$ Meehl ${ }^{4}$ extended the concept of schizotypy, proposing that a genetically determined defect in neural function invariably results in the development of a set of schizotypal features. Schizotypal features were later considered to be common features in the general population and were considered related to character traits and cognitive style. Recently, it has 
been considered that schizotypy results from an interaction between a schizophrenic genotype and environmental factors. 5,6

The concept of schizotypy has been considered as multidimensionally similar to schizophrenia. ${ }^{7}$ The Schizotypal Personality Questionnaire (SPQ) rating of schizotypy was developed in accordance with the criteria for schizotypal personality disorder (SPD) in the Diagnostic and Statistical Manual of Mental Disorders (Third Edition, Revised $[D S M-I I I-R]){ }^{8}$ SPD, as defined by the DSM-III-R, is similar to schizophrenia, but it does not meet the diagnostic criteria for schizophrenia. The validity of the SPQ as a screening tool for SPD has been confirmed, and some patients with a high SPQ score present symptoms consistent with the diagnostic criteria for SPD. ${ }^{9}$ The SPQ is made up of three factors: (1) the "cognitive-perceptual factor" includes the items concerning ideas of reference, odd beliefs or magical thinking, and unusual perceptual experiences; (2) the "interpersonal factor" includes the items concerning excessive social anxiety and no close friends; and (3) the "disorganized factor" includes the items concerning constricted affect, odd or eccentric behavior, and odd speech.

OCD patients have a high score on cognitive-perceptual and disorganized factors of schizotypy in comparison with other anxiety disorder patients. ${ }^{10}$ Moreover, the correlation between schizotypy and the severity of obsessive-compulsive symptoms (OCSs) has been consistently found in university students and OCD patients. ${ }^{1-14}$ Enright and Beech ${ }^{15}$ have explored the links between schizotypy and OCD by utilizing experimental paradigms drawn from cognitive science. The authors found a task designed to investigate inhibitory mechanisms in selective attention showed that OCD patients were different from other anxiety disorder patients. OCD patients exhibited a non-inhibition response pattern very similar to that previously observed in schizotypy and schizophrenia patients. ${ }^{16}$

Relationships between schizotypy and specific OCSs have also been reported. Tallis and Shafran ${ }^{14}$ showed that schizotypy was correlated with impulse, precision, and rumination OCSs rather than washing and checking OCSs. The authors suggested that the reduced cognitive inhibition observed in both schizotypy and OCD may result in the frequent entry into awareness of unacceptable urges and intrusive thoughts.

Therefore, the present authors hypothesized that schizotypy was correlated with the severity of obsession rather than the severity of compulsion. In the present study, the authors examined the relationship between the severity of obsession or compulsion and schizotypy to verify this hypothesis. Furthermore, the authors investigated the relationship between the severity of obsession or compulsion and schizotypal factors of the SPQ.

\section{Methods \\ Subjects}

A total of 61 OCD outpatients in the University Hospital at the Kyoto Prefectural University of Medicine (Kyoto, Japan) during the period 2008-2010 were recruited as subjects for the study. The patients were primarily diagnosed with OCD in accordance with the criteria of the DSM-IV-R ${ }^{17}$ All subjects were diagnosed using the Structured Clinical Interview for $D S M-I V-R$ Axis I disorders. Exclusion criteria included schizophrenia, neurological disorders, or a head injury. One patient met the criteria of schizophrenia and was therefore excluded from the study. The final number of OCD outpatients enrolled in the study was 60 (25 males and 35 females). Two patients had comorbidities that included major depressive disorders, two had specific phobias, one had social anxiety disorder, and 55 had OCD as their sole diagnosis. With regard to medication, 13 patients were not taking any drugs and 47 subjects were taking selective serotonin reuptake inhibitors. None of the subjects were exposed to cognitive behavioral therapy. All procedures were approved by the Kyoto Prefectural University of Medicine Research Ethics Committee (approval number c-379) before the study was undertaken. After a description of the study was given to all subjects, the authors obtained consent in writing from each patient.

\section{Assessment}

The authors used the Japanese version of the Yale-Brown Obsessive Compulsive Scale (Y-BOCS), which comprises ten items (five items for obsession, five items for compulsion) used to measure the severity of OCSs. ${ }^{18}$ Each item was rated on a five-point scale (ranging from 0 to 4 ), and a total score could range from 0 to 40 points.

The Japanese version of the SPQ is a self-completed questionnaire comprising 74 items. ${ }^{19}$ As mentioned earlier, the SPQ is made up of three factors: (1) the cognitive-perceptual (33 items); (2) the interpersonal (25 items); and (3) the disorganized factors (16 items). The form of response is "Yes" or "No." An item that is answered "Yes" is rated 1 point, and a total score could range from 0 to 74 points.

The Japanese version of the Hamilton Depression Rating Scale (HAM-D) is a structured interview comprising 21 items used to evaluate depression..$^{20} \mathrm{~A}$ total score could range from 0 to 63 points. 
The Japanese version of the Hamilton Anxiety Rating Scale (HAM-A) is a structured interview comprising 14 items used to evaluate anxiety. ${ }^{21}$ Each item was rated on a five-point scale (ranging from 0 to 4 ), and a total score could range from 0 to 56 points.

\section{Statistics}

The Pearson correlation coefficients between the Y-BOCS and SPQ scores were estimated so as to evaluate the relationship between OCS severity and three schizotypal factors. Hierarchical multiple linear regression analyses were then conducted to assess whether schizotypy predicted the severity of obsession and compulsion. In these models, the total score of SPQ was entered as an independent variable and the scores of obsession and compulsion on the Y-BOCS were entered as dependent variables. Lewandowski et $\mathrm{al}^{22}$ reported that schizotypy was associated with both depression and anxiety. Therefore, the total HAM-D and HAM-A scores were entered to control for the effect of depression and anxiety in the first step of the analysis, and the total SPQ score was entered in the second step. All analyses were conducted using SPSS software (v 17.0 for Windows; SPSS Inc, Chicago, IL).

\section{Results}

Table 1 shows the descriptive statistics of the demographic and clinical variables. The mean OCS severity was 24.2 , as measured by the Y-BOCS total score. The mean depression (measured by the HAM-D) and anxiety scores (measured by the HAM-A) were 7.8 and 8.0, respectively, and both depression and anxiety were mild.

Table 2 shows the Pearson correlation coefficients between the subindicators of the Y-BOCS and the SPQ. The Y-BOCS total score was correlated with the SPQ cognitive-perceptual $(r=0.34, P=0.008)$ and disorganized factors $(r=0.28, P=0.02)$. The Y-BOCS obsession score also was correlated with the SPQ cognitive-perceptual $(r=0.36$,

Table I Demographic and clinical characteristics $(n=60)$

\begin{tabular}{ll}
\hline Characteristic & Results, mean (SD) \\
\hline Age (years) & $32.9(9.8)$ \\
Education (years) & $13.7(2.0)$ \\
Y-BOCS total score & $24.2(6.5)$ \\
Y-BOCS obsession score & $12.0(3.5)$ \\
Y-BOCS compulsion score & $12.2(3.6)$ \\
HAM-D score & $7.8(4.5)$ \\
HAM-A score & $8.0(5.3)$ \\
SPQ score & $24.7(13.6)$ \\
\hline
\end{tabular}

Abbreviations: SD, standard deviation; Y-BOCS, Yale-Brown Obsessive Compulsive Scale; HAM-D, Hamilton Depression Rating Scale; HAM-A, Hamilton Anxiety Rating Scale; SPQ, Schizotypal Personality Questionnaire.
Table 2 Pearson correlation coefficients between Yale-Brown Obsessive Compulsive Scale (Y-BOCS) and Schizotypal Personality Questionnaire (SPQ) scores

\begin{tabular}{llll}
\hline SPQ score & \multicolumn{4}{l}{ Y-BOCS score, $\boldsymbol{r}(\boldsymbol{P})$} \\
\cline { 2 - 4 } & Total & Obsession & Compulsion \\
\hline Total & $0.31^{*}(0.02)$ & $0.34^{* *}(0.009)$ & $0.24(0.06)$ \\
Cognitive-perceptual & $0.34^{* *}(0.008)$ & $0.36^{* *}(0.005)$ & $0.24(0.07)$ \\
Interpersonal & $0.21(0.34)$ & $0.21(0.34)$ & $0.10(0.42)$ \\
Disorganized & $0.28^{*}(0.02)$ & $0.3 I^{*}(0.01)$ & $0.20(0.11)$ \\
\hline
\end{tabular}

Notes: $* P<0.05 ; * * P<0.01$.

$P=0.005)$ and disorganized factors $(r=0.31, P=0.01)$. By contrast, the Y-BOCS compulsion score had no correlation with either SPQ subscales. Moreover, The SPQ interpersonal factor had no correlation with either Y-BOCS subscales.

The authors then performed the hierarchical multiple linear regression analyses to evaluate whether schizotypy predicted the severity of obsession and compulsion. Table 3 shows the result of linear regression analysis predicting the Y-BOCS obsession score. The SPQ total score was a significant predictor of the Y-BOCS obsession score after accounting for control variables $\left(\beta=0.26\right.$, adjusted $R^{2}=0.09, \mathrm{~F}=2.84$, $P=0.04)$. On the other hand, the SPQ total score did not predict the Y-BOCS compulsion score after accounting for control variables $(\mathrm{F}[3,55]=0.18$; not significant). In addition, Table 4 indicates Pearson correlation coefficients between dependent variables for linear regression analyses. A correlation was found between the HAM-D and the HAM-A.

\section{Discussion}

\section{Correlation between the severity of OCSs and schizotypy}

The results of this study showed the correlation between the severity of OCSs and schizotypy in OCD patients. This result is consistent with findings from a previous study of OCD

Table 3 Linear regression analysis predicting the Yale-Brown Obsessive Compulsive Scale obsession score

\begin{tabular}{lllllll}
\hline Dependent variable & B & SEs & $\boldsymbol{\beta}$ & $\boldsymbol{r}$ & $\boldsymbol{R}^{2}$ & Adjusted $\boldsymbol{R}^{2}$ \\
\hline Step I & & & & & & \\
$\quad$ HAM-D score & 0.09 & 0.12 & 0.12 & 0.22 & 0.07 & 0.04 \\
$\quad$ HAM-A score & 0.12 & 0.10 & 0.18 & 0.25 & & \\
Step 2 & & & & & & \\
$\quad$ HAM-D score & 0.13 & 0.12 & 0.11 & 0.22 & 0.13 & $0.09 *$ \\
HAM-A score & 0.15 & 0.10 & 0.12 & 0.25 & & \\
SPQ score & 0.07 & 0.32 & $0.26 *$ & 0.31 & & \\
\hline
\end{tabular}

Note: $* P<0.05$.

Abbreviations: HAM-D, Hamilton Depression Rating Scale; HAM-A, Hamilton Anxiety Rating Scale; SPQ, Schizotypal Personality Questionnaire; B, regression coefficient; SE standard error; $\beta$, standard partial regression coefficient; $r$, correlation coefficient. $R^{2}$, decision coefficient. 
Table 4 Pearson correlation coefficients between dependent variables for linear regression analyses

\begin{tabular}{llll}
\hline & HAM-D score & HAM-A score & SPQ score \\
\hline HAM-D score & $\mathrm{I}$ & & \\
HAM-A score & $0.58^{*}(0.00 \mathrm{I})$ & $\mathrm{I}$ & \\
SPQ score & $0.17(0.20)$ & $0.25(0.06)$ & $\mathrm{I}$ \\
\hline
\end{tabular}

Note: $* P<0.01$.

Abbreviations: HAM-D, Hamilton Depression Rating Scale; HAM-A, Hamilton Anxiety Rating Scale; SPQ, Schizotypal Personality Questionnaire.

patients. ${ }^{14}$ Although these previous studies used OCS rating scales other than the Y-BOCS (eg, the Padua Inventory, the Maudsley Obsessive-Compulsive Inventory [MOCI], and the Obsessive-Compulsive Inventory), these studies showed the same results - the correlation between the severity of OCSs and schizotypy in OCD patients. ${ }^{11-14}$ It is worth noting here that the clinician-rated Y-BOCS is more objective than selfcompleted questionnaires.

\section{Conclusion}

In the present study, the authors report that the Y-BOCS obsession score, not the Y-BOCS compulsion score, was correlated with the SPQ total score. To the best of the authors' knowledge, this is the first study investigating the relationship between schizotypy and OCS severity in OCD patients by separating OCS severity into subcategories of obsession and compulsion. Y-BOCS obsession score was the significant predictor of SPQ total score. Since HAM-D and HAM-A didn't predict SPQ total score, it was the authors' belief that this result was not affected by depression and anxiety. As the authors hypothesized, the results of this study show that schizotypy was correlated with the severity of obsession rather than the severity of compulsion. This finding suggests that OCD patients with an elevated SPQ total score experience a reduction of cognitive inhibition, resulting in the frequent entry into obsession. In the correlations between subscales of Y-BOCS and SPQ, the Y-BOCS total score was correlated with the SPQ cognitive-perceptual and disorganized factors. This result is consistent with the findings of Roth and Baribeau, ${ }^{11}$ which indicate that the MOCI total score is correlated with the SPQ cognitive-perceptual and disorganized factors. Furthermore, the results of the present study show the detailed relationships by classifying OCSs into obsession and compulsion. To be specific, the Y-BOCS obsession score had correlations with the SPQ cognitive-perceptual and disorganized factors but no correlation with the interpersonal factor. Compulsion had no correlation with either SPQ subscales. This suggests that obsession is not indefinitely correlated with schizotypy but, rather, that it is correlated with specific schizotypal factors (eg, cognitive-perceptual and disorganized factors). Regarding the correlation between the severity of obsession and the SPQ cognitive-perceptual factor, since the items of the cognitive-perceptual factor include the idea of reference and magical thinking, a patient with a severe obsession may be likely to have a bizarre and incorrect confidence (ie, a delusion). Additionally, regarding the correlation between the Y-BOCS obsession score and the SPQ disorganized factor, since the items of the disorganized factor include bizarre appearance and talking, a patient with severe obsession may be likely to be disturbed and frequently filled with obsession, resulting in bizarre talking. By contrast, the SPQ interpersonal factor was interestingly not correlated with either Y-BOCS subscales. This finding was consistent with the results of Roth and Baribeau's study, ${ }^{11}$ which also did not indicate a correlation between MOCI total score and the SPQ interpersonal factor. The items of the SPQ interpersonal factor include social anxiety and emotional repression. Some OCD patients force or persuade their family and/or others to confirm or ensure their OCSs (ie, involvement). Patients who involve others are generally highly dependent and poor at suppressing emotion (ie, immature type), which may have led to the results in this study.

\section{Limitations and future tasks}

Since this was a cross-sectional study, it was impossible to explain the effect of schizotypy on the clinical course of OCD, for example. Therefore, future longitudinal studies are recommended to clarify this by examining the changes of schizotypy along the clinical course of OCD patients.

\section{Disclosure}

The authors report no conflicts of interest in this work.

\section{References}

1. National Comorbidity Survey [webpage on the Internet]. National Comorbidity Survey (NCS) and National Comorbidity Survey Replication (NCS-R). Boston, MA: Harvard School of Medicine; 2005. Available from: http://www.hcp.med.harvard.edu/ncs/. Accessed August 4, 2012.

2. Sobin C, Blundell ML, Weiller F, Gavigan C, Haiman C, Karayiorgou M. Evidence of a schizotypy subtype in OCD. J Psychiatr Res. 2000;34(1): $15-24$.

3. Rado S. Dynamics and classification of disordered behavior. Am J Psychiat. 1953;110(6):406-416.

4. Meehl PE. Schizotaxia, schizotypy, schizophrenia. Am Psychol. 1962;17(2):827-838.

5. Claridge G. Theoretical Background and Issue. Oxford: Oxford University Press; 1997.

6. Claridge G, Davis C. Personality and Psychological Disorders. London: Arnold; 2003.

7. Raine A, Reynolds C, Lencz T, Scerbo A, Triphon N, Kim D. Cognitiveperceptual, interpersonal, and disorganized features of schizotypal personality. Schizophr Bull. 1994;20(1):191-201. 
8. American Psychiatric Association. Diagnostic and Statistical Manual of Mental Disorders, 3rd ed, revised. Washington, DC: American Psychiatric Press; 1987.

9. Raine A. The SPQ: a scale for the assessment of schizotypal personality based on DSM-III-R criteria. Schizophr Bull. 1991;17(4):555-564.

10. Claridge G, Broks P. Schizotypy and hemisphere function: I. Theoretical considerations and the measurement of schizotypy. Pers Indiv Differ. 1984;5(6):633-648.

11. Roth RM, Baribeau J. The relationship between schizotypal and obsessive-compulsive features in university students. Pers Indiv Differ. 2000;29(6):1083-1093.

12. Lee HJ, Telch MJ. Autogenous/reactive obsessions and their relationship with OCD symptoms and schizotypal personality features. J Anxiety Disord. 2005;19(7):793-805.

13. Lee HJ, Cougle JR, Telch MJ. Thought-action fusion and its relationship to schizotypy and OCD symptoms. Behav Res Ther. 2005; 43(1):29-41.

14. Tallis F, Shafran R. Schizotypal personality and obsessive compulsive disorder. Clin Psychol Psychot. 1997;4(3):172-178.

15. Enright SJ, Beech AR. Reduced cognitive inhibition in obsessivecompulsive disorder. Br J Clin Psychol. 1993;32(Pt 1):67-74.

16. Laplante L, Everett J, Thomas J. Inhibition through negative priming with Stroop stimuli in schizophrenia. Br J Clin Psychol. 1992;31(Pt 3): 307-326.
17. American Psychiatric Association. Diagnostic and Statistical Manual of Mental Disorders, 4th ed, revised. Washington, DC: American Psychiatric Press; 2000.

18. Nakajima T, Nakamura M, Taga C, et al. Reliability and validity of the Japanese version of the Yale-Brown Obsessive-Compulsive Scale. Psychiatry Clin Neurosci. 1995;49(2):121-126.

19. Iijima Y, Sasaki J, Bando N, Asai T, Mouri I, Tanno Y. Development of a Japanese version of the Schizotypal Personality Questionnaire and factor structure of schizotypy. Jpn J Behav Ther. 2010;36(1): 29-41.

20. Nakane Y, editor. Japanese Version of Structured Interview Guide for the Hamilton Depression Rating Scale. Tokyo: Seiwa-Shoten; 2004.

21. Otsubo T, Kamijima K. [Japanese version of Hamilton Anxiety Rating Scale Interview guide]. Jpn J Clin Psychopharmacol. 2005;8: 1588-1593. Japanese.

22. Lewandowski KE, Barrantes-Vidal N, Nelson-Gray RO, Clancy C, Kepley HO, Kwapil TR. Anxiety and depression symptoms in psychometrically identified schizotypy. Schizophr Res. 2006;83(2-3): $225-235$.
Neuropsychiatric Disease and Treatment

\section{Publish your work in this journal}

Neuropsychiatric Disease and Treatment is an international, peerreviewed journal of clinical therapeutics and pharmacology focusing on concise rapid reporting of clinical or pre-clinical studies on a range of neuropsychiatric and neurological disorders. This journal is indexed on PubMed Central, the 'PsycINFO' database and CAS.

\section{Dovepress}

The manuscript management system is completely online and includes a very quick and fair peer-review system, which is all easy to use. Visit http://www.dovepress.com/testimonials.php to read real quotes from published authors. 